FOLIA SCANDINAVICA VOL. 18 POZNAŃ 2015 DOI: $10.1515 / \mathrm{fsp}-2015-0017$

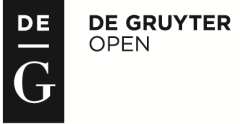

PRESSto. IMU. Adam Mickiewicz University Repository

\title{
ADJEKTIVE MIT NEGATIONSPRÄFIXEN IM HEUTIGEN DÄNISCH
}

\author{
EUGENIUSZ RAJNIK
}

Adam Mickiewicz University in Poznań

\begin{abstract}
The article offers a classification of adjective prefixes in Danish (section 3) including, among others, native and foreign prefixes (e.g. gen- and $a$-, respectively), genuine prefixes (e.g. agte-) and particle prefixes (e.g. anti-). Various definitions of prefixal derivatives have been presented in section 4 , while section 5 shows examples of and discusses native and foreign negative prefixes modifying the meanings of adjectives in contemporary Danish. The adjectives in question have been analyzed with regards to their structure and the emotional load they convey. Section 6 concludes the paper and includes comments on the productivity of the prefixes in question, as well as synonymy and antonymy in their usage.
\end{abstract}

\section{EINLEITUNG}

Im vorliegenden Artikel wird versucht, zu einem besseren Verständnis des Wesens der Adjektive mit Negationspräfixen im Dänischen beizutragen. In der Darstellung soll eine synchron-strukturelle Analyse der genannten Bildungen vorgenommen werden. Der Beitrag setzt sich zum Ziel, die Rolle dieser Wortbildungstypen im Sprachsystem zu analysieren und Kriterien für ihre Klassifikation zu erarbeiten.

Meine Aufgabe war es, auf einige Probleme und Tendenzen hinzuweisen, die für den Sprachwissenschaftler und Sprachlehrer von Bedeutung sind. Die Kenntnis der Wortbildungsmodelle, zu denen man u. a. adjektivische Präfixableitungen zählt, ermöglicht bessere Einsichten in die Struktur der Wörter. Sie gehören zu den produktiven Wortbildungsarten im heutigen Dänisch. Es wird versucht, eine Typisierung der adjektivischen Bildungen mit einheimischen und fremden Negationspräfixen im Dänischen vorzunehmen. Ich werde mich hauptsächlich auf produktive Modelle konzentrieren und will zeigen, wie sie entstanden sind. Dabei geht es um die Aufdeckung von 
existierenden Wortbildungsregeln, die der Bildung der untersuchten Strukturen zugrunde liegen und die Grundlage für neue Präfixbildungen liefern. Diese Analyse liefert einen Ausgangspunkt für die Formulierung einiger Regeln und Restriktionen morphologischer und semantischer Art in Bezug auf die oben genannten Präfixableitungen.

\section{ZUR MATERIALAUSWAHL}

Der vorliegende Beitrag basiert auf einer Sammlung von Beispielen, die in überwiegender Zahl aus den dänischen Standardwörterbüchern (u. a. Politikens Nudansk Ordbog med etymologi B. udg. 2005/ und Den Danske Ordbog /2003-2005/) stammen. Diesen Wörterbüchern wurden auch Angaben zur Etymologie entnommen.

Außerdem wurde die (nicht umfangreiche) wissenschaftliche Literatur über den Gebrauch der adjektivischen Präfixbildungen im Dänischen verwendet, vor allem Pia Jarvads Nye ord - hvorfor og hvordan? (1995) und Aage Hansens Moderne Dansk II (1967), aber manche adjektivische Präfixableitungen, die darin belegt sind, sind nicht mehr aktuell, d.h. sie sind heute bereits veraltet bzw. nicht mehr oder nur selten gebräuchlich. Das in den Quellen Vorgefundene wurde auch auf Grund der eigenen Sprachkompetenz kritisch geprüft.

Es wurde angestrebt, eine relativ repräsentative Auswahl von Beispielen zu gewinnen, um die erörterten Probleme näher zu beleuchten. Die Liste der Beispiele ist ohne Schwierigkeit erweiterbar (besonders mit dem Präfix $u$-), aber die Einbeziehung einer größeren Anzahl von Beispielen in die Untersuchung hätte zu keinen wesentlich anderen Ergebnissen geführt. Bei der Auswahl von Einzelbeispielen wird das Schwergewicht auf diejenigen Präfixbildungen gelegt, die sich bei synchroner Betrachtungsweise analysieren lassen. Im Beschreibungsteil sind auch solche Konkurrenzformen (bei gleicher Basis) miteinbezogen, zwischen denen keine semantische Differenzierung besteht, die sich aber in ihrer Gebrauchsfrequenz unterscheiden, z. B. umoralsk (167) - amoralsk (75). In Klammern wurde die Anzahl der Okkurrenzen im KorpusDK angegeben. Außerdem werden solche Formen berücksichtigt, die semantisch unterschiedlich sind, z. B. usocial $\neq$ antisocial.

\section{EINTEILUNG VON ADJEKTIVISCHEN PRÄFIXEN}

In morphologischer Hinsicht sind Präfixe gebundene Morpheme, die vor einer Basis auftreten. Sie kommen nicht als selbständige Wörter vor. In derivierten Adjektiven determinieren in der Regel Präfixe das Basiswort 
(vgl. Donalies, 2005:107), z. B. hyperkorrekt 'sehr korrekt', usikker, urgammel. Sie dienen der Bildung expliziter Derivate und können in der Regel die Wortart nicht verändern, vgl. autoritcer - antiautoritcer, traditionel - utraditionel. Man unterscheidet zwischen einheimischen Präfixen (z. B. gen-, mis-, sam-, u-, ur-, van-, arke-) und Fremdpräfixen (z. B. a-, de-, dis-, in-, mega-) (vgl. Rajnik, 2011:104). Manche Präfixe sind betont (z. B. mis- in 'misforståelig, 'mistroisk), andere unbetont (z. B. $a$ - in ate istisk). Sie verbinden sich mit Substantiven, z. B. genbrug, megatilbud, Adjektiven, z. B. urgammel, Verben, z. B. begå, bibeholde, und Adverbien, z. B. uheldigvis. Einige Präfixe (z. B. mis-) sind mit allen drei Hauptwortarten kombinierbar, z. B. mistolkning, misforståelig, misbruge. Es gibt jedoch auch solche einheimischen Präfixe (z. B. bein bemoerke), die fast ausschließlich an verbale Basen treten.

Man unterscheidet auch zwischen echten Präfixen, z. B. anti-, gen-, mis-, ko-, non-, u-, die nie abtrennbar sind, und Partikelpräfixen, z. B. over-, under, die „freie homonyme Entsprechungen“ (Römer, 2006:32) haben und in der Regel den Hauptakzent tragen, z. B. 'underjordisk. Präfixe kommen vor allem in Modifikationsbildungen vor, sie können ein Basislexem grammatisch und semantisch modifizieren. Hansen/Heltoft (2011:249) unterscheiden zwischen echten (z. B. be- in beskrive), festen (z. B. på- in påminde) und freien (z. B. ind-in indsatte, vgl. satte ind) Präfixen.

Es gibt ein begrenztes Inventar an Präfixen, aber es können immer wieder mal neue hinzukommen. Die Reihenbildung ist unterschiedlich, da die produktiven Präfixe offensichtlich Distributionsbeschränkungen unterliegen. Nur einige der in Frage kommenden Präfixe begegnen uns häufiger (besonders $u$-). Es ist nicht ohne Bedeutung, welches Präfix sich mit welchem Basisadjektiv verbindet. Wo mehrere Bildungsweisen und Präfixe zur Verfügung stehen, gibt es keine völlige Beliebigkeit der Anwendung, sondern gewisse Gebrauchsbeschränkungen. Zwar sind $u$ - und ir- weitgehend austauschbar (vgl. ureligiфs /4/ - irreligiфs /10/), wenn wir die unterschiedliche Frequenz außer Acht lassen. So lässt sich z. B. $a$ bzw. $u$ - nicht durch non- ersetzen, und es gibt bestimmte distributionelle Unterschiede zwischen diesen Präfixen, vgl. ateistisk : *nonteistisk, umoralsk: *nonmoralsk. Nur wenige Präfixe sind so produktiv, dass sie in großem Ausmaß reihenbildend wirken. Es ist vor allem das Negationspräfix $u$-, aber nicht jedes Adjektiv scheint mit $u$ - kombinierbar $\mathrm{zu}$ sein, und $u$ - unterliegt offensichtlich Gebrauchsbeschränkungen, vgl. *utroende - ikketroende (als Kompositum), wo die Negationspartikel ikke- eingesetzt wird. 


\section{AUFFASSUNGEN VON ADJEKTIVISCHEN PRÄFIXBILDUNGEN}

Die Präfixbildungen entstehen durch Kombination eines Wortstamms mit einem Präfix, und sie weisen eine Bedeutungsnuance auf, nämlich Modifikation (vgl. Kessel/Reimann, 2008:106). Die Erweiterung und Modifikation der Lexeme erfolgt mit Hilfe von Präfixen, die im Dänischen ererbt sein können oder griechischen bzw. lateinischen Ursprungs sind. Letztere haben sich im Dänischen eingebürgert, sind aber teilweise auf die Verbindung mit fremdwörtlichen Ableitungsbasen griechischer oder lateinischer Herkunft beschränkt. Für manche Präfixableitungen, besonders mit solchen Präfixen wie anti-, eks-, pseudo-, super-, sind in der Regel englische Muster maßgebend (ähnlich wie im Deutschen, vgl. Erben, 2006:111) gewesen. Manche englische Präfixbildungen sind als Modelle für Analogiebildungen aufzufassen.

Präfixale Bildungen sollen folgende Kriterien erfüllen:

1) Der Wortstamm, der meistens eine Ableitung ist, kommt in freier Verwendung vor, vgl. antisemitisk : semitisk. Unberücksichtigt bleiben hier solche Bildungen wie z. B. nonchalant (vgl. nonchalance) : *chalant, dyslektisk (vgl. dysleksi) : *lektisk, weil das zweite Glied als selbständiges Wort nicht existiert.

2) Es liegt ein klarer Motivationsbezug der Präfixbildung auf den Wortstamm vor, d.h. die gleiche Bedeutung ist in beiden vorhanden. Außer Betracht lasse ich im Folgenden die sogenannten Präelemente (meistens lateinischer oder griechischer Herkunft), die bereits in der Basis enthalten sind und sich im Dänischen nur auf diachronischer Ebene segmentieren lassen, z. B. konin konstruktiv (vgl. konstruere, dekonstruktiv). Synchronisch gesehen gehören solche Konstruktionen nicht zu Präfixbildungen, sondern stellen Derivate von entsprechenden präfigierten Basisverben dar.

\section{ADJEKTIVISCHE PRÄFIXBILDUNGEN MIT EINHEIMISCHEN UND FREMDSPRACHLICHEN NEGATIONSPRÄFIXEN}

Negationspräfixe kommen bei Adjektiven häufiger als bei Substantiven vor. Bei den adjektivischen Präfixbildungen wird das Basislexem umgebildet und semantisch modifiziert. In der folgenden Tabelle gebe ich eine Übersicht über die Arten der Modifikation der Basis (vgl. Erben, 2006:111). Dabei werden die Negationspräfixe in bestimmte Bedeutungsgruppen eingeordnet, 
da sich mit jedem Präfix ein bestimmtes semantisches Merkmal verbindet. Die negierenden einheimischen Präfixe und Fremdpräfixe sind vor allem an der Konstitution der folgenden Wortbildungsbedeutungen beteiligt:

\begin{tabular}{|c|c|c|c|}
\hline $\begin{array}{c}\text { ART DER } \\
\text { MODIFIKATION } \\
\text { DER BASIS } \\
\end{array}$ & $\begin{array}{c}\text { ZUSÄTZLICHE } \\
\text { SEMANTISCHE } \\
\text { MERKMALE } \\
\end{array}$ & $\begin{array}{l}\text { EINHEIMISCHE } \\
\text { PRÄFIXE }\end{array}$ & BEISPIELE \\
\hline \multirow[t]{2}{*}{ Negation } & $\begin{array}{l}\text { a. 'falsch' } \\
\text { b. 'nicht' } \\
\text { c. 'verkehrt' }\end{array}$ & $\begin{array}{l}\text { mis- } \\
u- \\
\text { van- }\end{array}$ & $\begin{array}{l}\text { misinformeret } \\
\text { usikker } \\
\text { vanhellig }\end{array}$ \\
\hline & & $\begin{array}{c}\text { FREMDSPRACHLICHE } \\
\text { PRÄFIXE }\end{array}$ & \\
\hline Negation & $\begin{array}{l}\text { a. 'nicht, gegen' } \\
\text { b. 'nicht' }\end{array}$ & $\begin{array}{l}\text { anti- } \\
\text { kontra- } \\
\text { a- } \\
\text { an- } \\
\text { de- } \\
\text { des- } \\
\text { dis- } \\
\text { dys- } \\
\text { in- } \\
\text { il- } \\
\text { im- } \\
\text { ir- } \\
\text { non- }\end{array}$ & $\begin{array}{l}\text { antikommunistisk } \\
\text { kontrarevolutionar } \\
\text { ateistisk } \\
\text { analfabetisk } \\
\text { decentral } \\
\text { desinformeret } \\
\text { disharmonisk } \\
\text { dysfunktionel } \\
\text { inaktiv } \\
\text { illegal } \\
\text { impotent } \\
\text { irrationel } \\
\text { nonverbal }\end{array}$ \\
\hline
\end{tabular}

(Tabelle 1) Übersicht über die Arten der Modifikation der Basis.

Ich wende mich nun der Darstellung einheimischer und fremdsprachlicher Negationspräfixe im Einzelnen zu. Die meisten von ihnen (mit einigen Beispielen) sind bereits bei Hansen (1967 II:447-451) in den Abschnitten „Hjemlige præfikser” und „Fremmede præfikser” vorhanden. Es muss bemerkt werden, dass die Distribution der einheimischen Präfixe (mis-, $u$-, van-) nicht so stark eingeschränkt ist wie die der Fremdpräfixe, die sich hauptsächlich mit fremdwörtlichen Basen verbinden. Die Kriterien der Beschreibung sind das jeweilige Präfix und seine Funktion (die im Präfix enthaltenen Merkmale). Es kann nicht die Aufgabe dieser Darstellung sein, auf die Präfixe in aller Ausführlichkeit einzugehen, deswegen wurden nur besonders typische Beispiele herangezogen. Die anschließende Übersicht über die Typen von negierenden Präfixableitungen im modernen Dänisch erhebt keinen Anspruch auf Vollständigkeit und berücksichtigt nur die Fakten, die für die Analyse der oben genannten Konstruktionen relevant sind. Der folgende Überblick ist alphabetisch nach den einzelnen einheimischen Präfixen und Fremdpräfixen geordnet. 


\subsection{ADJEKTIVISCHE PRÄFIXBILDUNGEN MIT EINHEIMISCHEN NEGATIONSPRÄFIXEN}

\subsubsection{DAS PRÄFIX MIS-}

Das Präfix mis- (< altnord. mis-, missi- < gemeingerm. *missa- 'verkehrt') ist (wie im Deutschen, vgl. Donalies, 2005:27) mit Substantiven, Adjektiven und Verben kompatibel. Es bezeichnet das Verkehrte oder Verfehlte, also die Abweichung vom Normalen, z. B. mismodig, mistolket, mistrøstig, und es verbindet sich mit Adjektiven positiven Inhalts. Negative Empfindung und Wertung hat es u. a. in misbrugt. Wie im Deutschen (vgl. Fleischer/Barz, 1995:201) drückt mis- bloße Negation nicht aus, vgl. misfarvet - ufarvet, misforståelig - uforståelig. Adjektive und partizipiale Adjektive mit mis- haben in der Regel einen Hauptton auf dem Präfix, z. B. 'mislykket, 'misvisende, mitunter auf dem Wortbildungsstamm, z. B. mis'undelig, aber in Einzelfällen treten beide Varianten auf: 'mismodig oder mis'modig. Dieses Präfix war im Dänischen schon früher gebräuchlich. Mis- verbindet sich nicht mit adjektivischen Simplizia, sondern wird mit Adjektiven kombiniert, die eine andere Struktur aufweisen, das sind u. a. deverbale Derivate, z. B. mistcenkelig (:mistcenke), misundelig (:misunde), und desubstantivische Derivate, z. B. mistroisk (:mistro). Explizite Derivate kommen auch als Basis vor, z. B. misfornøjet (:fornøjet). Oft sind das konvertierte Adjektive aus Partizip-IIFormen (vgl. Donalies, 2007:91), z. B. misinformeret, mistolket.

In der Bedeutung berühren sich die Bildungen mit dem Präfix miszum Teil mit dem Präfix van- bzw. mit fejl-, vgl. misrøgtet (15) (Part. Perf.) vanrøgtet (7) (Part. Perf.), mistolket (9) - fejltolket (3). Manchmal kommt es zu Konkurrenzen mit unterschiedlichem Grad von Synonymie - mit demselben Stamm, aber mit einem anderen Suffix, vgl. mistankelig (276) (z. B. mistcenkelige personer) - mistcenksom (212) (z. B. en mistcenksom tolder), oder mit demselben Stamm, aber die zweite Form als Adjektivkonvertat mit partizipialem Zweitglied, vgl. misforståelig (4) (z. B. et misforståeligt udtryk) - misforstået (506) (z. B. misforstået loyalitet). In manchen Fällen konkurrieren mis-Bildungen mit Adjektiven mit dem Halbsuffix -løs, vgl. mistrøstig (48) - trøsteløs (4). Antonymisch zu mis- ist ret-, vgl. misvisenderetvisende.

\subsubsection{DAS PRÄFIX U-}

Das Präfix $u$ - (< altnord. ú-, $o$-, altengl. un-, lat. in-, griech. $a(n)$-), das nur als Nominalpräfix (beim Adjektiv und Partizipialadjektiv stärker genutzt als beim Substantiv) vorkommt, hat sich sehr stark ausgebreitet und ist semantisch gefächert. Die Basiswörter bezeichnen oft psychische Zustände bzw. Verhaltensweisen von Personen. Viele Negationsbildungen sind von hoher Frequenz, jedoch scheint nicht jedes Adjektiv mit $u$ - kombinierbar zu sein. 
Das Präfix $u$ - hat eine feste Bedeutung und ist ein Ausdruck für die Negation. Konträre Klassen bezeichnen u. a. uartig, uansvarlig, ufin, umild, uopdragen, usand, usikker, utro, utcet, uvis (vgl. Hansen, 1967 II:449), aber bei manchen Adjektiven wird die negative Wertung des $u$-Adjektivs abgeschwächt, vgl.

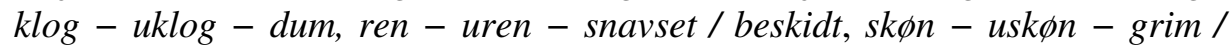
hasslig (ähnlich wie im Deutschen, vgl. Fleischer/Barz, 1995:272, Eisenberg, 2004:248ff.). Mitunter hat $u$ - die Wertung 'vom Normalen zum Falschen hin abweichend', z. B. uvillig. In einzelnen Fällen begegnet $u$ - bei Mengenbezeichnungen in augmentativer Funktion, z. B. utallig (z. B. utallige gange).

Es tritt hauptsächlich mit Ableitungsbasen lateinischer, z. B. uprofessionel, oder griechischer, z. B. udemokratisk, aber auch altnordischer, z. B. utryg, deutscher, z. B. uangribelig, französischer, z. B. uelegant, und norwegischer, z. B. uhyggelig, Herkunft auf. Adjektive mit $u$ - haben gewöhnlich einen Hauptton auf dem Präfix, z. B. 'uklar, 'ucegte, 'usand, 'uskarp, aber mitunter auf dem Wortbildungsstamm, z. B. uan'steendig, uan'vendelig. In der Regel ist es sinnvoller, positiv konnotierte Formen zu negieren als pejorativ gewertete durch eine Negation positiv zu machen. Das Präfix $u$ - ist verbindbar vor allem mit Adjektiven, die positiv Bewertetes bezeichnen (ähnlich wie das Präfix un- im Deutschen, vgl. Erben, 2006:113), z. B. ubehagelig, uhøflig, upopular, upålidelig, uvenlig, uvenskabelig. Es gibt einzelne Fälle, wo eben das Basisadjektiv selbst negativ konnotiert wird, vgl. bureaukratisk - ubureaukratisk, dфdelig - udфdelig, farlig - ufarlig, skyldig uskyldig. Bei einigen Präfixableitungen mit $u$ - ist die potentielle Basis als freies Adjektiv gegenwärtig nicht gebräuchlich, vgl. uafvargelig *afvargelig, ubrydelig - *brydelig, ubandig - *bandig, uforskammet *forskammet, usmagelig - *smagelig, ustandselig - *standselig, utallig *tallig (vgl. Hansen, 1967 II:449). Das Präfix $u$-verbindet sich sowohl mit abstrakten Adjektiven, z. B. uheldig, ulykkelig, als auch mit konkreten, z. B. umenneskelig. Es kann sowohl mit einfachen Adjektiven, z. B. udansk, ufed, ufrom, ujavn, ulig, usund, als auch mit komplexen Adjektivstämmen, z. B. u(i)genkendelig, urolig, urytmisk, utilstrakkelig, vorkommen. Die Verbindung mit komplexen Adjektivstämmen ist häufiger. Manchmal sind Präfixbildungen, z. B. ubebygget, ubekendt, uundgåelig, oder Komposita mit Adjektiv als Basis geläufig, z. B. uegennyttig, ufaglart, ufuldkommen, uselvstcendig, uarbejdsdygtig (44) (auch arbejdsudygtig (1) - ohne Präfix $u$-, aber mit dem Infix - $u$-). Sehr häufig sind Adjektive mit partizipialer Basis (Partizip-II-Formen), z. B. uadresseret, uafgjort, uafhentet, uafklaret, uafsluttet, uelsket, usminket. Das Partizip II erhält adjektivischen Charakter, z. B. uafbrudt arbejde. Relativ selten kommen Adjektive aus Partizip-IFormen vor, z. B. uanende, ucharmerende, udeltagende, uforstående, ufyldestgфrende, utiltalende. 
In Einzelfällen steht $u$ - neben $a$-, dis-, in-, ir- bzw. ikke- und mod- bei gleicher Basis mit semantischer Differenzierung, manchmal ohne wesentliche Unterschiede, vgl. uharmonisk (20) - disharmonisk (24), uhistorisk (26) ahistorisk (23), umoralsk (167) - amoralsk (75), unormal (292) - anormal (3), upolitisk (92) - apolitisk (19) - ikkepolitisk (2) / ikke-politisk (5), ureligiøs (4) - irreligiфs (10), usymmetrisk (2) - asymmetrisk (75), uvillig (105) - modvillig (292), aber: usocial (3) (abwertend, z. B. usocial politik) - asocial (188) (abwertend, z. B. asocial opførsel). In solchen Fällen könnte man von „funktionalen Abstufungen“ (Erben, 2006:114) sprechen.

Eine Anzahl von $u$-Bildungen treten in Konkurrenz zu anderen Bildungen (mit demselben Stamm, aber mit einem anderen Suffix oder mit dem Partizipmorphem - $/ e / t$ ), aber in den meisten Fällen lassen sich zwischen ihnen semantische Unterschiede nachweisen, vgl. ubehjcelpelig (14) - ubehjalpsom (69), uantastelig (15) - uantastet (98), ubestridelig (126) - ubestridt (101), udefinerbar (57) - udefinerlig (37), uerkendelig (5) - uerkendt (15). Manche Adjektive mit $u$ - haben Synonyme (ohne Präfix und Suffix, aber mit dem Halbsuffix -lфs bzw. -fri), vgl. uansvarlig (378) - ansvarsl $\phi s$ (74), uendelig (1748) - endel фs (549), ufejlbarlig (76) - ufejlbar (1) - fejlfri (195), uformel (424) - informel (13) - formlфs (30), uinteressant (354) - interessel $\phi s$ (14), ukarakteristisk (25) - karakterlфs (18), ukritisk (272) - kritiklфs (57), uproblematisk (132) - problemlфs (26) - problemfri (177), usmagelig (150) smaglфs (80) - smag(s)fri (5), utro (267) - trolфs (146), utrøstelig (44) trøstelфs (4). In der Bedeutung berühren sich die Präfixbildungen mit $u$ - zum Teil mit denen mit anti-, vgl. usocial (usocial politik) $\neq$ antisocial (antisocial adfard). Differenzierungen (mit demselben Stamm, aber mit einem anderen Suffix, oder mit dem Partizipmorphem -le/t bzw. mit dem Halbsuffix -l $\phi s$ und ohne Präfix) zeigen u. a. ubeboelig (en ubeboelig ejendom) $\neq$ ubeboet (en ubeboet $\emptyset)$, ubeskrivelig $\neq$ ubeskrevet, ubetalelig $\neq$ ubetalt, ubetonkelig $\neq$ ubetonksom, ubrugelig $\neq$ ubrugt, uformel $\neq$ uformelig, uformelig $\neq$ forml $\phi$ s, utålelig $\neq$ utålsom (selten). Antonymisch wird manchmal vel- verwendet, vgl. uanvendt - velanvendt, uargumenteret - velargumenteret, uartikuleret velartikuleret, ubegavet - velbegavet, ubegrundet - velbegrundet, ubekendt velbekendt, ubeskyttet - velbeskyttet, udefineret - veldefineret.

\subsubsection{DAS PRÄFIX VAN-}

Das Präfix van- (< altnord. van-, altengl. wan- abgeleitet von einem Adj., altnord. vanr 'mangelnde', verwandt mit lat. vanus 'leer') ist an der Negation beteiligt. Das Modell ist heute nur schwach produktiv (vgl. Skautrup, 1968 IV:250, Hansen, 1967 II:449). Es kommt bei Adjektiven seltener als bei Substantiven vor. Dieses Präfix kann sich mit einem einfachen, z. B. vantro, oder einem komplexen Wortbildungsstamm verbinden, z. B. vanartet. 
Es drückt einen konträren Gegensatz aus, z. B. vanhellig, vanskabt, vantro. Das Präfix van- wird teilweise mit mis- synonymisch gebraucht, vgl. vanrøgtet (7) (Part. Perf.) - misrøgtet (15) (Part. Perf.).

\subsection{ADJEKTIVISCHE PRÄFIXBILDUNGEN MIT FREMDSPRACHLICHEN NEGATIONSPRÄFIXEN}

\subsubsection{DIE PRÄFIXE A-/AN-}

Das Präfix $a$ - (< griech. $a$ - 'un-, nein, nicht') hat die Variante $a b$-, z. B. abnorm (unormal), oder an- vor einem Vokal, z. B. analfabetisk, anorganisk (selten). Es kommt beim Adjektiv häufiger als beim Substantiv vor und tritt mit Ableitungsbasen griechischer oder lateinischer Herkunft auf. Es drückt einen kontradiktorischen Gegensatz aus, z. B. abiotisk, agnostisk, amusisk, aseksuel, asynkron, ateistisk, atypisk (vgl. Hansen, 1967 II:450). Pejorative Funktion hat das Präfix $a$ - u. a. in amoralsk.

In der Bedeutung berühren sich die Bildungen mit dem Präfix $a$ - zum Teil mit denen mit $u$-, anti- oder ikke-, vgl. ahistorisk (23) - uhistorisk (26), amoralsk (75) - umoralsk (167), anorganisk (2) - uorganisk (79), anormal (3) - unormal (292), aseptisk (18) - antiseptisk (13), apolitisk (19) - upolitisk (92) - ikkepolitisk (2) / ikke-politisk (5), aperiodisk (3) - ikke-periodisk (1), asymmetrisk (75) - usymmetrisk (2), asocial (188) (abwertend, z. B. asocial opførsel) - usocial (3) (abwertend, z. B. usocial politik). Differenzierungen von $a$ - und anti- zeigen u. a. asocial (usocial politik) $\neq$ antisocial (antisocial adfard).

\subsubsection{DIE PRÄFIXE ANTI-/ANT-}

Das Präfix anti- (< griech. anti 'gegen, wider, im Gegenteil') ist im Dänischen unter englischem Einfluss seit dem 19. Jh. produktiv, besonders in Presse und Literatur. Die Präfixvariante ant- erscheint nur vor einem Vokal, z. B. antarktisk (vgl. Hansen, 1967 II:450). Das Präfix anti- drückt einen kontradiktorischen Gegensatz aus und tritt heute hauptsächlich mit fremder Basis auf, z. B. antiautoritcer, antidepressiv, antiklerikal, antizionistisk, aber manchmal auch mit einheimischer Basis, z. B. antiborgerlig, antikirkelig. Dieses Präfix verbindet sich u.a. mit von Länder- und Völkernamen abgeleiteten Adjektiven, z. B. antiisraelsk, antijødisk, manchmal stößt man auf solche Formen in der Sprache der Politik, z. B. antidemokratisk, und im medizinischen Fachvokabular, z. B. antibakteriel, antitoksisk. In vielen Fällen tritt es mit Basen (meistens auf -isk) auf, deren Bedeutung sich auf eine negativ bewertete Eigenschaft bezieht, vgl. fascistisk - antifascistisk, auch: antiimperialistisk, -kommunistisk, -militaristisk, -nazistisk, -racistisk, -totalitcer. Es gibt aber Fälle, wo eben das Basisadjektiv positiv oder neutral 
und die Präfixbildung negativ bewertet ist, vgl. antiintellektuel, -parlamentarisk, -semitisk. Bei einigen Präfigierungen mit anti- ist die potentielle Basis als freies Adjektiv gegenwärtig nicht gebräuchlich, vgl. antipatisk (vgl. antipati) : *patisk, antikonceptionel (vgl. antikonception) : *konceptionel, antitetisk (vgl. antitese) : *tetisk.

Einer Anzahl von Präfixbildungen stehen zur gleichen Basis gebildete konkurrierende Parallelformen mit unterschiedlichem Grad von Synonymie gegenüber, vgl. antireligiфs (6) - areligiфs (3), antiseptisk (13) - aseptisk (18), aber: antisocial $\neq$ asocial (abwertend) - usocial (abwertend). Das Präfix antidrückt das Entgegengesetzte in Bildungen mit pro- aus, vgl. antiamerikanskproamerikansk, antiarabisk - proarabisk, antivestlig - provestlig.

\subsubsection{DAS PRÄFIX DE-}

Das Präfix $d e-(<$ lat. $d \bar{e}-$ 'ab-, ent-') ist beim Adjektiv selten und drückt das Gegenteil von etwas aus und erscheint in Adjektiven oder Partizipialadjektiven mit fremder (meist lat.) Basis, z. B. decentral, deformeret, degraderet, dekonstruktiv, demonteret, dereguleret, destabiliseret. Manchmal konkurriert de- mit af-, vgl. demilitariseret (8) - afmilitariseret (6).

\subsubsection{DAS PRÄFIX DES-}

Das Präfix des- (< franz. dés- < lat. dis- 'auseinander, un-') ist beim Adjektiv schwach entwickelt. Es erscheint vor einem Vokal und impliziert negative Empfindung des Adjektivs (als Ausnahme gilt desinficeret), und alle Partizipialadjektive mit des- verbinden sich mit fremder Basis, z. B. desinteresseret, desorienteret.

\subsubsection{DAS PRÄFIX DIS-}

Das Präfix dis- (< lat. dis- 'auseinander, un-') begegnet selten in Adjektiven oder Partizipialadjektiven mit fremder Basis und drückt einen kontradiktorischen oder konträren Gegensatz aus, z. B. diskvalificeret, disproportional. Synonymisch dazu teilweise $u$-, vgl. disharmonisk (26) uharmonisk (20), auch, aber mit dem Partizipmorphem -t: diskontinuerlig (11) - diskontinuert (3).

\subsubsection{DAS PRÄFIX DYS-}

Das Präfix dys- (< griech. dys- 'übel, schlecht, miss-') tritt mit Adjektiven nur vereinzelt auf und dient dazu, eine Fehlfunktion auszudrücken, z. B. dysfunktionel. 


\subsubsection{DIE PRÄFIXE IN-/IL-/IM-/IR-}

Die Präfixe il-/im-/ir- 'nicht' sind positionsbedingte Varianten von in(< lat., entspricht und ist verwandt mit griech. $a / n /-$ ), die durch Assimilation an den folgenden Konsonanten entstanden sind: vor $m$, $p$ erscheint im-, z. B. immobil, impotent, vor $l$-il-, z. B. illegal, illegitim, illoyal, und vor $r-i r-$, z. B. irrelevant (vgl. Hansen, 1967 II:450, Hansen, 2001:15). Diese Präfixe kann man phonologisch und semantisch als Allomorphe betrachten. Sie kommen beim Adjektiv häufiger als beim Substantiv vor. Sie können sich vor allem mit einem mehrsilbigen fremdwörtlichen Adjektiv verbinden, z. B. inaktiv, indirekte, indiskutabel, ineffektiv, infinit, inhabil, inhuman, immateriel, instabil, intolerant, oder mit partizipialem Zweitglied, z. B. indisponeret. In Einzelfällen sind nur die Formen mit in- üblich, vgl. inappellabel - *appellabel. Das Präfix in- (auch il-, -im und ir-) hat nur verneinende Funktion und drückt in der Regel einen konträren Gegensatz aus, z. B. indefinit, indiskret, ineffektiv, inkompatibel, inkompetent, inkonvertibel, inkongruent, inkonsekvent, intransitiv, auch: illitteroer, irregulaer.

Manchmal kommt es zu Konkurrenzen (in-/ir- vs. $u$-) mit unterschiedlichem Grad von Synonymie, vgl. informel (13) - uformel (424), irreligiфs (10) ureligiøs (4), auch (mit einem anderen Suffix): irrationel (218) - irrational (9), z. B. irrationalt tal. In solchen Fällen drücken die Bildungen mit $u$ - einen kontradiktorischen Gegensatz aus (ähnlich wie im Englischen, vgl. Hansen et al., 1982:78).

\subsubsection{DAS PRÄFIX KONTRA-}

Das Präfix kontra- (< lat. contra- 'gegen, entgegengesetzt') ist selten vertreten und bringt meist eine Gegenüberstellung zum Ausdruck, z. B. kontrafaktisk, kontraintuitiv, kontraproduktiv, kontrarevolutionaer. Es verbindet sich vor allem mit Adjektiven, die lateinischer Herkunft sind. Das Modell ist heute nur schwach produktiv.

\subsubsection{DAS PRÄFIX NON-}

Das Präfix non- (< lat. non- 'nicht') bildet kein produktives Modell im Dänischen. Adjektive mit non- haben gewöhnlich einen Hauptton auf dem Präfix, z. B. 'nonfigurativ. Es hat verneinende Funktion und drückt einen kontradiktorischen Gegensatz aus (ähnlich wie im Englischen, vgl. Hansen et al., 1982:80), z. B. nonverbal. Im Gegensatz zu ikke- kommt non- nur in einzelnen Bildungen vor Wörtern fremder Herkunft vor, z. B. nonfigurativ, nonverbal (non + verbal /Adjektiv/, vgl. verbal als Substantiv). Bei einigen 
Präfigierungen mit non- ist die potentielle Basis als freies Adjektiv gegenwärtig nicht gebräuchlich, vgl. nonchalant : *chalant. Synonymisch dazu teilweise ikke-, vgl. nonverbal (36) - ikke-verbal (2).

\section{SCHLUSSFOLGERUNGEN}

Abschließend lässt sich Folgendes feststellen:

1) Nicht alle Präfixe sind in der gleichen Weise reihenbildend geworden. Die am meisten ausgebaute Gruppe sind die Negationsbildungen mit anti-, in-, mis- und vor allem $u$-. Die Zahl der Bildungen mit anderen Pejorativpräfixen ist nicht groß oder sehr gering. Manche Typen scheinen nicht produktiv zu sein.

2) Einige Präfixe unterliegen einer regelmäßigen Angleichung an den Anlaut des Basislexems, z. B. il- (vor -l-) / im- (vor - m-) / ir- (vor - $r$-).

3) Manche Präfixe können synonymische oder antonymische Beziehungen eingehen. So konkurrieren synonymisch u. a. $a$-, ir- und $u$-, z. B. umoralsk (167) - amoralsk (75), unormal (292) - anormal (3), irreligiøs (10) - ureligiøs (4). Zwischen den beiden Formen gibt es im Grunde genommen keine semantische Differenzierung. Sie sind weitgehend austauschbar, aber der Unterschied liegt in den kontextuellen Komponenten (vgl. et misforståeligt udtryk - misforstået loyalitet) und in der Gebrauchsfrequenz. Bei manchen Präfixbildungen ist eine außerordentlich häufige Anwendung zu bemerken. Bei der Antonymie handelt es sich meistens um eine konträre Gegensatzbeziehung, vgl. antiisraelsk-proisraelsk.

4) Für mehrere Arten der Modifikation der Basis stehen mehrere Präfixe zur Verfügung. Die Präfixbildungen mit den Negationspräfixen, wie $a$-, anti-, il-, in-, ir-, non- und $u$-, drücken gewöhnlich einen kontradiktorischen oder einen konträren Gegensatz aus und stehen $\mathrm{zu}$ ihren Basen im Verhältnis der Komplementarität, d.h. es werden Gegensätze polarer Art gebildet, vgl. rationel - irrationel, teistisk - ateistisk.

5) Eine Anzahl von Präfixbildungen treten in Konkurrenz zu anderen Bildungen, aber in einigen Fällen lassen sich zwischen ihnen semantische Unterschiede nachweisen, vgl. antisocial $\neq$ asocial.

6) Es werden immer noch neue verneinende Präfixableitungen im Dänischen gebildet, z. B. dekonstruktiv, useriфs. Viele von ihnen sind formal an entsprechende lateinische bzw. griechische Ableitungen angelehnt, die vor allem im Fachvokabular entstanden waren und als Vorbild für Analogiebildungen im Dänischen wirkten. Dort hatten sie ein formales Gegenstück in einem präfixlosen Adjektiv. 


\section{LITERATUR}

Den Danske Ordbog. 2003-2005. Hovedredaktører Ebba Hjorth og Kjeld Kristensen. Bind 1-6. Udg. af Det Danske Sprog- og Litteraturselskab. København: Gyldendal. http://ordnet.dk/ddo

Donalies, E. (2005). Die Wortbildung des Deutschen. Ein Überblick. 2. überarbeitete Aufl. Tübingen: Narr.

Donalies, E. (2007). Basiswissen Deutsche Wortbildung. Tübingen und Basel: A. Francke.

Eisenberg, P. (2004). Grundriss der deutschen Grammatik. Band 1: Das Wort. 2., überarb. und aktual. Aufl. Stuttgart: Metzler.

Erben, J. (2006). Einführung in die deutsche Wortbildungslehre. 5., durchgesehene und ergänzte Aufl. Berlin: E. Schmidt Verlag.

Fleischer, W., Barz, I. (1995). Wortbildung der deutschen Gegenwartssprache. 2. durchgesehene und ergänzte Aufl. Tübingen: Max Niemeyer Verlag.

Hansen, Aa. (1967). Moderne Dansk. Bind II. København: Grafisk Forlag.

Hansen, B. (et al.). (1982). Englische Lexikologie. Einführung in Wortbildung und lexikalische Semantik. Leipzig: VEB Verlag Enzyklopädie.

Hansen, E. (2001). Damonernes Port. Støttemateriale til undervisningen i nydansk, 4. udg. København: Hans Reitzels Forlag.

Hansen, E., Heltoft, L. (2011). Grammatik over det Danske Sprog. Bind I. København: Det Danske Sprog- og Litteraturselskab (DSL). Odense: Syddansk Universitetsforlag.

Jarvad, P. (1995). Nye ord - hvorfor og hvordan? København: Gyldendal.

Kessel, K., Reimann, S. (2008). Basiswissen Deutsche Gegenwartssprache. 2. überarbeitete Aufl., Tübingen und Basel: A. Francke Verlag.

KorpusDK http://ordnet.dk/korpusdk

Politikens Nudansk Ordbog med etymologi. (2005). 3. udg. København: Politikens Forlag.

Rajnik E. (2011). Substantivische Derivate mit Fremdpräfixen im Dänischen. Scripta Neophilologica Posnaniensia. Tom XI, 103-121.

Römer, Ch. (2006). Morphologie der deutschen Sprache. Tübingen und Basel: A. Francke Verlag.

Skautrup, P. (1968). Det danske Sprogs Historie. Bind IV. København: Nordisk Forlag.

\section{Eugeniusz Rajnik}

Uniwersytet im. Adama Mickiewicza w Poznaniu

Katedra Skandynawistyki

al. Niepodległości 4

61-874 Poznań

Poland

rajnik@amu.edu.pl 